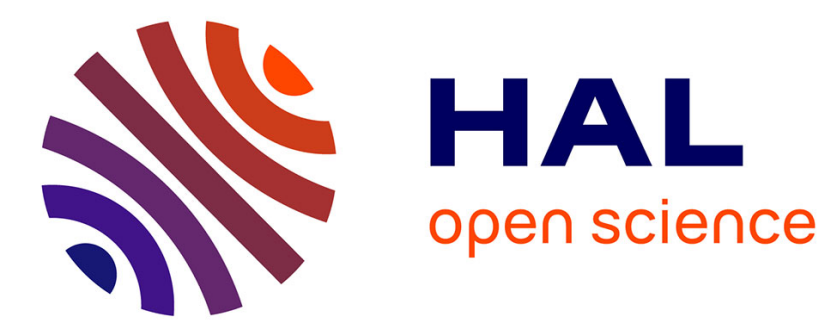

\title{
Characterizing the Influence of Motion Parameters on Performance when Acquiring Moving Targets
}

Alexandre Kouyoumdjian, Nicolas Ferey, Patrick Bourdot, Stéphane Huot

\section{To cite this version:}

Alexandre Kouyoumdjian, Nicolas Ferey, Patrick Bourdot, Stéphane Huot. Characterizing the Influence of Motion Parameters on Performance when Acquiring Moving Targets. 15th Human-Computer Interaction (INTERACT), Sep 2015, Bamberg, Germany. pp.148-155, 10.1007/978-3-319-227238_12. hal-01207511

\author{
HAL Id: hal-01207511 \\ https://hal.inria.fr/hal-01207511
}

Submitted on 1 Oct 2015

HAL is a multi-disciplinary open access archive for the deposit and dissemination of scientific research documents, whether they are published or not. The documents may come from teaching and research institutions in France or abroad, or from public or private research centers.
L'archive ouverte pluridisciplinaire HAL, est destinée au dépôt et à la diffusion de documents scientifiques de niveau recherche, publiés ou non, émanant des établissements d'enseignement et de recherche français ou étrangers, des laboratoires publics ou privés. 


\title{
Characterizing the Influence of Motion Parameters on Performance when Acquiring Moving Targets
}

\author{
Alexandre Kouyoumdjian ${ }^{1}$, Nicolas Férey ${ }^{1}$, Patrick Bourdot ${ }^{1}$, Stéphane Huot ${ }^{2}$ \\ ${ }^{1}$ Venise - LIMSI/CNRS, Orsay, France; ${ }^{2}$ INRIA, Lille, France
}

\begin{abstract}
Current pointing techniques provide no adequate way to select very small objects whose movements are fast and unpredictable, and theoretical tools -such as Fitts' law- do not model unpredictable motion. To inform the design of appropriate selection techniques, we studied how users performed when selecting moving objects in a 2D environment. We propose to characterize selection performance as a function of the predictability of the moving targets, based on three parameters: the speed $(S)$ of the target, the frequency $(F)$ at which the target changes direction, and the amplitude $(A)$ of those direction changes. Our results show that for a given speed, selection is relatively easy when $A$ and $F$ are both low or high, and difficult otherwise.
\end{abstract}

Keywords. pointing, picking, mobile targets, selection

\section{Introduction}

Interactive systems often rely on animated content and thus require the ability to select moving targets in a rapid and reliable way. For example, scientists working on a molecular dynamics simulation would need to isolate one or several moving particles to study their properties. "Freezing" the animation is a straightforward solution to this problem, but this is not applicable when the movement itself matters (e.g. molecular simulations or games) or when interacting with live data (e.g. air-traffic control).

Several efficient and promising techniques have been proposed to ease the selection of moving targets. For instance, Hook [8] relies on an effective target-prediction heuristic, but is not based on any particular theoretical analysis of moving targets. Comet or Target Ghost [5] rely on assumptions based on Fitts' law [3] in order to improve the effective width of the moving target or to reduce the pointing distance. However, it is not yet clear if this predictive model is appropriate.

While the relevance of Fitts' law to pointing tasks for moving targets has been investigated by psychologists [6,7], they mostly focused on movements along a straight path or in a regular and relatively predictable way. In interactive systems, however, selectable targets can move in a more erratic and unpredictable fashion (e.g. Brownian motion in molecular simulations, the ball in a video of a ball sport). A better understanding of how the nature of targets' motion affects pointing performance is there-

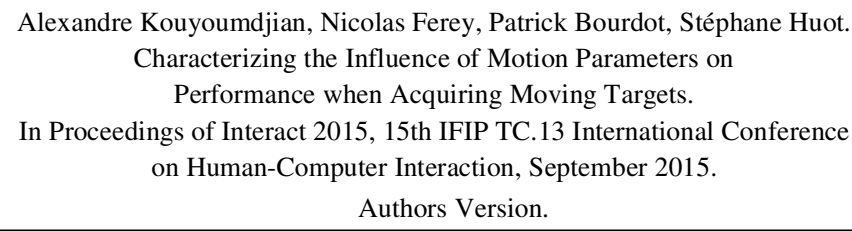


fore critical to inform the design of appropriate interaction techniques and to evaluate them as well. Since Fitts' width and amplitude fall short of fully characterizing the task of mobile target acquisition, we propose in this paper to consider also their speed and direction (frequency of changes and angles) as a way to characterize a broader range of motion, from regular to erratic.

We describe these parameters in the following section, as well as an experiment we conducted in order to assess users' performance when varying these parameters.

\section{Studying the Acquisition of Erratic Targets}

\subsection{Characterizing Erratic Motion}

In order to study performance when acquiring targets with erratic movements, we need a quantifiable, controllable and replicable way to generate such motion. We propose to characterize the nature of motion with three parameters:

- S: the speed at which the target moves, in $\mathrm{cm} / \mathrm{s}$;

- F: the frequency at which the target's direction vector is rotated, in $\mathrm{Hz}$;

- A: the maximum angle by which the direction vector of the target can be rotated: after every period $\mathrm{T}=1 / \mathrm{F}$, the direction vector of the target is rotated by the angle alpha which is pseudo-randomly sampled from [-A;+A] (with A in degrees of arc).

These parameters (and their values in the following experiment) only capture a part of all possible types of motion, since simulating all of them would have been too complex and unmanageable, with too many parameters (e.g., acceleration, curvature, etc.). We rather chose a trade-off between simplicity and the potential to simulate types of motion that participants in our experiment would perceive as fundamentally different in nature. In particular, varying $F$ and $A$ allows the simulation of a wide range of movements, from regular to erratic.

\subsection{Experiment}

We conducted an experiment with 13 right-handed participants, aged 14 to 56 . They were asked to click with a mouse on a moving target displayed on a desktop monitor.

Apparatus. The input device was a standard mouse without acceleration, moving a standard crosshair pointer on a Dell UltraSharp U2412M 24" display. The task was performed in a square window of 1000 pixels (about $23.87 \mathrm{~cm}$ ).

Task \& Conditions. The task consisted in selecting one circular target among 50, with a fixed diameter of $5.4 \mathrm{~mm}$. The 49 "distractors" were displayed in gray, and the target to select in red (see Figure 3(b)). Both the target and the distractors moved according to the current condition, defined by the $S, F$ and $A$ parameters, and bounced off the edges of the window. To control the pointing amplitude, the motion of the distractors was constrained so that after selecting a target, the distance between the 
mouse cursor and the next target was constant (of about $7.96 \mathrm{~cm}$ ). When the participant successfully selected the red target, it briefly turned green while the next target to select turned red at the same time.

Selection involves a trade-off between speed and accuracy [4], and users can adopt different approaches to this trade-off. In order to minimize such variation in selection strategy, participants were instructed to select the target as fast as possible but with at most 5 attempts. The number of remaining attempts for each trial was displayed in the top left corner of the window. After the last try, the word "failure" was displayed and the participant was asked to keep trying to select the target, but without trying to minimize errors. Thus, failures are not absolute, but indicate critical conditions for which selection is very difficult.

We tested all combinations of the following $(S, A, F)$ values, replicated 4 times:

- $S: 0.73,1.46$ and $2.19 \mathrm{~cm} / \mathrm{s}$ (3 values);

- $F: 1,2,4,8,13,20$ and $30 \mathrm{~Hz}$ (6 values);

- $A$ : $0,30,60,90,120$ and 180 degrees of arc (7 values).

An additional baseline condition was added with static targets, which was replicated 20 times. When $\mathrm{A}=0$, targets move in straight lines. In summary, we tested a total of $3 \times 6 \times 7+1=127$ conditions, and 524 trials $(126 \times 4+1 \times 20)$ per participant.

Procedure \& Data Collection. Each session lasted approximately 40 minutes, including a training phase of over 50 trials, after which participants felt comfortable with the task. Short breaks were allowed after $25 \%, 50 \%$ and $75 \%$ of the trials were completed. At the end of the session, participants were administered a short questionnaire about their perception of the different classes of motion and the strategies they had adopted.

We collected Selection Time from the moment the target turned red to the successful click, Erroneous Clicks (i.e. clicks outside of the target disk), and Failures (i.e. last erroneous clicks of the 5 tries).

\section{$3 \quad$ Results and Analysis}

\subsection{Selection Performance}

All the values presented here are averaged across the subjects. Performance in the easiest conditions was relatively stable across participants: our slowest participant was less than 4 times slower than the fastest one for straight motion. However, there was a considerable amount of variation for the most difficult conditions (by a factor of over 18 from the fastest to the slowest participant). We therefore normalized selection times to avoid bias from the slowest participants: for each participant, the resulting averages of normalized selection times (ANSTs) are the average of selection time per condition, normalized according to the most difficult condition for the given participant. Therefore, if a condition has an ANST of 0.75 for a given participant, it 
means that this participant needed $75 \%$ as much time on average to select targets in this condition as in the condition he/she found most difficult. Because the condition that was precisely the most difficult may be slightly different for each participant, no condition has an average ANST of 1.0 across all subjects.

The number of errors ranges from 0 to 5 . An error rate of 3.5 for a given condition means that on average, participants made 3.5 failed clicks for each trial in that condition. A failure rate of $50 \%$ for a given condition means that on average, $50 \%$ of the trials in that condition were failed, i.e. that participants made at least 5 failed clicks in $50 \%$ of their trials. Note that because of growing concerns in various research fields over the limits of null hypothesis significance testing for reporting and interpreting experimental results [1,2], our discussions are based on effect sizes (reported as percent differences ${ }^{1}$ ).

Speed. We observed that both selection time and error rate increase when speed increases, as illustrated in Figure 1(a): average ANST at the highest speed (37\%) is higher than in the lowest one (17\%) with a percent difference of $74 \%$. We observe that selection time and error rates are roughly affine functions of speed. We only included the plots for $A=90$, but the results for all other values of $A$ are very similar.

Angle. Parameter $A$ exhibits more complicated tendencies, with an interaction with both $S$ and $F$. At low speed, $A$ has a small effect on selection times (for $S=0.73$ and $F=4$, when $\mathrm{A}=0$, ANST $=14 \%$ and when $A=120$, ANST $=20 \%$; percent difference of $33 \%$, the highest observed at this speed) but a bigger effect at high speed (for $\mathrm{S}=2.19$, at the same $A$ and $F$, ANSTs are respectively $21 \%$ and $54 \%$; percent difference of $90 \%$ ). A possible explanation is that at low speed, selection is almost as easy as with static targets, regardless of the nature of the motion. Figure 1(c) illustrates the effect of A at high speed. We observe that as long as frequency is low, higher values of $A$ imply higher selection times. However, as $F$ increases, the effect of $A$ on selection time is changed: after steadily increasing with $A$, selection time reaches a plateau, and then decreases. Furthermore, the value of $A$ associated with this plateau $\left(A_{\text {peak }}\right)$ depends on $F$ : as $F$ increases, $A_{\text {peak }}$ decreases.

We also observed that the number of errors and failure rates follow the same trends and that at medium speed, these trends are similar but less pronounced. ${ }^{1}$ Percent differences between 2 measures $\left(\mathrm{m}_{1}, \mathrm{~m}_{2}\right)$ were computed using $p=100 \times \frac{\left(m_{2}-m_{1}\right)}{\frac{1}{2} \times\left(m_{1}+m_{2}\right)}$ in order to
get symmetric measures of relative differences. 


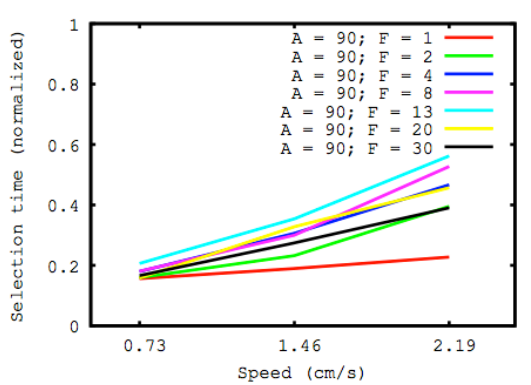

(a) ANST vs. $S$ for $A=90$.

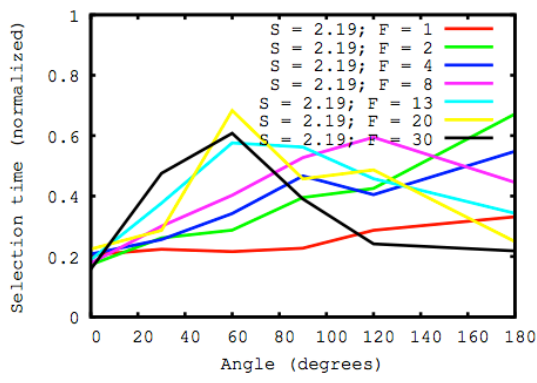

(c) ANST vs. $A$ for $S=2.19$.

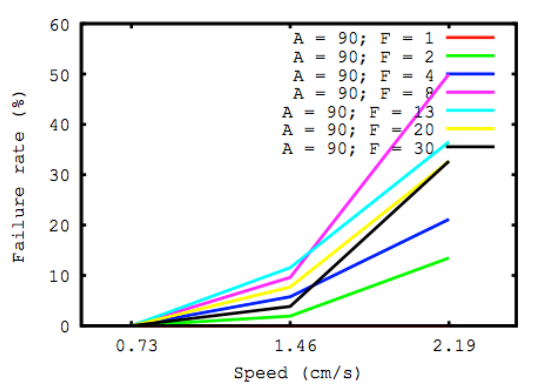

(b) Failure rates vs. $S$ for $A=90$.

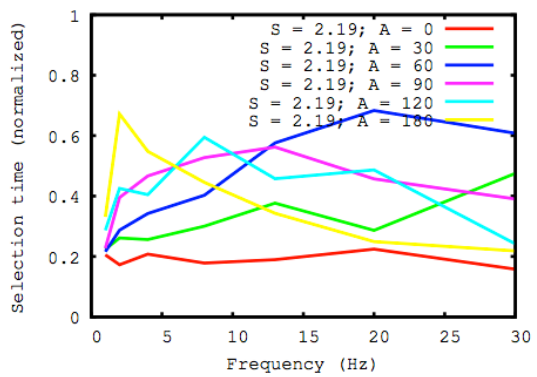

(d) ANST vs. $F$ for $S=2.19$.

Figure 1 - Selection performance as a function of $S, A$ and $F$.

Frequency. A similar pattern can be observed for frequency: $F$ has a smaller effect on selection time when speed is low, but a larger one at high speed. On Figure 1(d), we observe that for lower values of $A$, higher frequencies are correlated with higher selection time, but for higher values of $A$, the trend changes. When $A$ is high, selection time increases with $F$ up to a certain peak $\left(F_{\text {peak }}\right)$ and then decreases. Furthermore, the value of $F_{\text {peak }}$ decreases as $A$ increases. Trends for number of errors and failure rates are consistent with those observed for selection time, and as for angle widths, trends at medium speed are similar to those at high speed, although less pronounced.

\subsection{Exploratory Analysis}

We also considered our parameter space as a whole in order to identify general trends. The heat maps in figures 2(a) and 2(b) represent the ANSTs for medium and high speeds. In each of those heat maps, $A$ stands on the horizontal axis, and $F$ on the vertical one. Each intersection is thus the average of ANSTs for the corresponding condition. The heat maps in figures 2(b), 2(c) and 2(d) present the highest speed we tested, since it resulted in the most pronounced effects of $A$ and $F$. In Figure 2(b), we observe that a region of the heat map approximately centred on the main diagonal (from topleft to bottom-right) exhibits the highest selection times. Figure 2(c) displays the av- 
erage error rates for each condition, which are correlated with selection times. Finally, failure rates are presented in figure $2(\mathrm{~d})$.

These heat maps suggest that selection is especially difficult for combinations of wide angles with low frequencies, narrow angles with high frequencies, or medium angles with moderate frequencies.

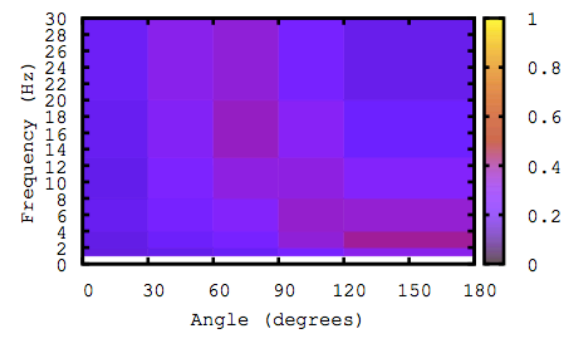

(a) ANST vs. $A$ and $F$ for $S=1.46$.

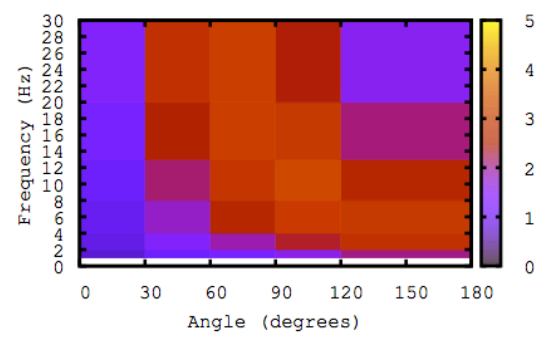

(c) Error rates (out of 5) for $S=2.19$.

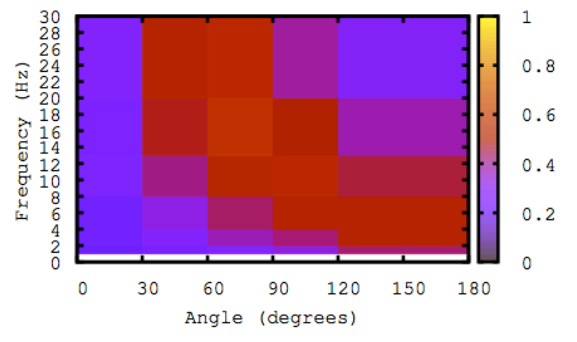

(b) ANST vs. $A$ and $F$ for $S=2.19$.

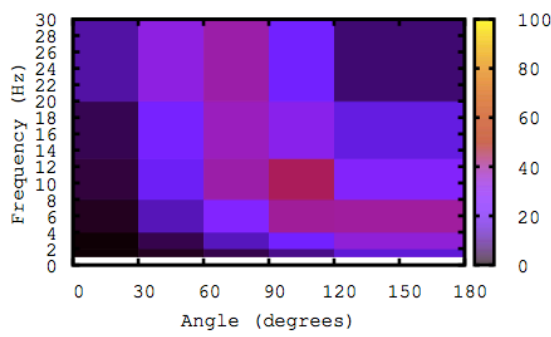

(d) Failure rates (in \%), for $S=2.19$.

Figure 2 - Selection performance as a function of $A$ and $F$, for different values of $\mathrm{S}$.

From Angle and Frequency to Predictability. The trends highlighted above suggest that the product $A \times F$ (angle width by frequency) might roughly predict selection performance for erratic targets. We plotted this product against selection time in figure 3 (a), and it reveals that an index of difficulty (or predictability) for irregular motion could be derived from $A \times F$. Furthermore, when $A \times F$ reaches a certain value $A F_{\text {peak }}$, which in our data is around 1,100 , selection time is maximal. We suppose that $A F_{\text {peak }}$ would vary in different conditions, e.g. target width or pointing distance as described by Fitts' law, but it remains stable for the three different speeds we have tested, and therefore probably independent of this parameter. As mentioned before, we observed error rate to be generally strongly correlated with selection time, and so to also depend on the $A \times F$ product. 
Subjective Impressions. The answers to our questionnaire were consistent with, and complementary to, our quantitative results. Indeed, $92 \%$ of our participants were able to identify different categories of motion: $77 \%$ of them identified at least three major categories, together with two or three corresponding selection strategies.

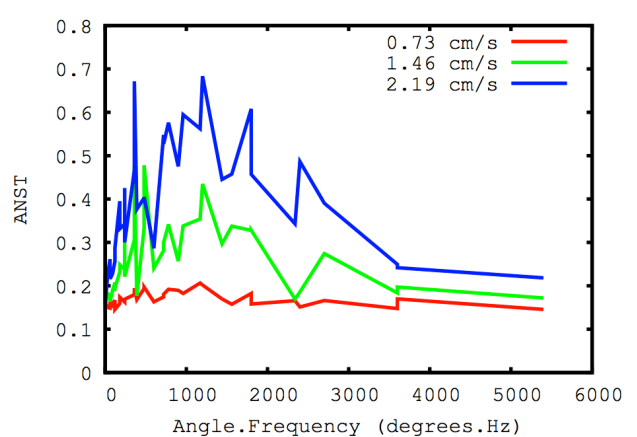

(a) ANST vs. $A F$ for all speeds.

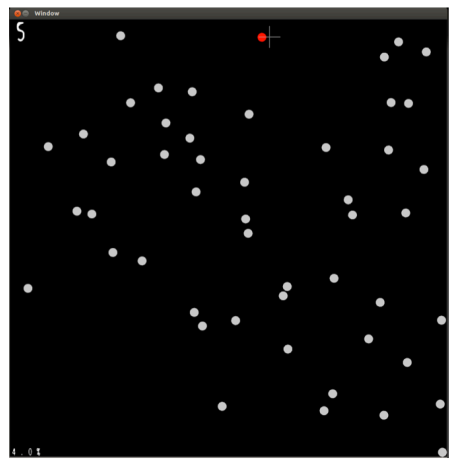

(b) Application used.

Figure 3 - Selection time as a function of $A \times F$. Screenshot of the application.

Categories and Strategies. Participants first described rather steady motion, in roughly straight lines, with few or small deviations. In the second case, they identified "jerky" or "erratic" motion that they characterized by frequent, significant changes in direction. In the third case, they described the motion as "Brownian", or "oscillations" or "vibrations". More interestingly, $92 \%$ of them explained that they would try to anticipate the target's movement and to intercept it, and those who had identified a steady category specifically linked this strategy to it. However, our participants had no "clever" way of catching jerky targets, and would simply try to be fast enough and click a lot, thus making many errors. For the vibrating category, 69\% of them said they would aim for the "average" position of the target over a short period of time, and either try to intercept it or just click and hope for the best.

Interpretation. The steady category reflects how our participants perceived the conditions where both angle width and change frequency were low. The jerky category relates to the difficult diagonal of our heat maps, and the vibrating category is the one with both high frequency and angle width. The important observation is that the strategy of anticipation requires a certain degree of predictability, since one can only anticipate what one can predict. Likewise, aiming for the average position of a target is a prediction. On the other hand, the jerky category seems to be very difficult to predict.

We therefore conclude that the steady and vibrating categories of moving targets are relatively easy to acquire because their movements are quite predictable, whereas jerky targets are more difficult because less predictable. Of course, the borders between these categories are hard to determine formally and are likely to depend on the user perception of the movement. 


\section{Conclusion and perspectives}

We have proposed a way to characterize the erratic behavior of moving targets with three parameters: speed, angle width and frequency, and tested pointing performance when varying these parameters. We have shown that the nature of target motion affects pointing performance, which is not captured by Fitts' law's index of difficulty. The latter can only describe the best case, and does not indicate that acquiring fast, highly erratic targets can be extremely difficult.

Although the $(S, A, F)$ set of parameters we have proposed models a continuum of target motion, our participants were able to distinguish at least three distinct categories that we have characterized (steady, vibrating and jerky). Our results suggest that for erratic targets, predictability determines pointing performance. And while the steady and vibrating categories are relatively predictable, the jerky one is not.

We have also observed that the $A \times F$ product might be a promising way to assess predictability, although we still need more data to deduce a predictive law for the acquisition of erratic targets. Nevertheless, this preliminary work is a first step toward a more formal study of the acquisition of erratic targets, and introduces a way to better characterize or even control their movement. Ultimately, such a model could be instrumental in the design of new selection techniques.

\section{Acknowledgements}

We wish to thank the participants for their time and effort.

\section{References}

1. P. Dragicevic, F. Chevalier, and S. Huot. Running an hci experiment in multiple parallel universes. In CHI 2014 Extended Abstracts, pp. 607-618. ACM, 2014.

2. G. Cumming. The new statistics: Why and how. Psychological science, 25(1):729, 2014.

3. Paul M Fitts. The information capacity of the human motor system in controlling the amplitude of movement. Journal of experimental psychology, 47(6):381, 1954.

4. Yves Guiard, Halla B Olafsdottir, and Simon T Perrault. Fitts' law as an explicit time/error trade-off. In Proc. of CHI 2011, pp. 1619-1628. ACM, 2011.

5. Khalad Hasan, Tovi Grossman, and Pourang Irani. Comet and target ghost: techniques for selecting moving targets. In Proc. of CHI 2011, pp. 839-848. ACM, 2011.

6. Errol R. Hoffmann. Capture of moving targets: a modification of Fitts' law. Ergonomics, 34(2):211-220, 1991

7. Richard J. Jagacinski, Daniel W. Repperger, Sharon L. Ward, and Martin S. Moran. A test of Fitts' law with moving targets. Human Factors: The Journal of the Human Factors and Ergonomics Society, 22(2):225-233, 1980.

8. Michael Ortega. Hook: Heuristics for selecting $3 \mathrm{~d}$ moving objects in dense target environments. In Proc. of 3DUI 2013, pp. 119-122. IEEE, 2013. 